\title{
LAS NOCIONES DE MANO, BRAZO Y CODO EN INDOEUROPEO
}

\begin{abstract}
The authoress studies the different names which have received the hand, the arm and the elbow in the Indo-European languages. She makes a comparative analysis of all the roots and stems which have originated such designations, in order to determine the antiquity and extensions of the extant formas.
\end{abstract}

Las partes del cuerpo constituyen un campo interesante dentro del vocabulario indoeuropeo, prestándose, además, a estudios lexicográficos de cierta garantía. Los nombres que han recibido algunas de estas partes en época indoeuropea resultan, a menudo, curiosos por proceder de usos onomatopéyicos o figurados. En otras ocasiones su denominación va ligada a raíces que designan la actividad que dicha parte tiene como misión principal ${ }^{1}$.

En algunos casos es posible rastrear cuál sería el sentido originario de la raíz de la que ha derivado una palabra. En otros, es imposible incluso el hallar la raíz originaria. La antigüedad de una denominación está señalada por varios factores, entre los que es primordial el hecho de que se encuentre documentada en tres o más lenguas indoeuropeas, a ser posible de áreas no relacionadas dialectalmente. Puede ocurrir que una raíz esté bien documentada en varias lenguas y, sin embargo, en cada una de ellas ha dado lugar a una denominación diferente. En este caso, habría que ver qué motivos han llevado a esta situación.

Por todo ello, nos hemos planteado este trabajo cuyo objeto es estudiar las denominaciones de la mano, el brazo y el codo en las lenguas indoeuropeas. Pretendemos ver, en primer lugar, si es posible reconstruir una única denominación para tales nociones, o si, por el contrario, nos hallamos ante casos aislados o de áreas dialectales más o menos

1 Cf. G. Bonfante, «Sull'animismo delle parti del corpo in indoeuropeo», $R L 4$, 1958 , p. 19 ss.

LIII, $2.0-4$ 
próximas. En segundo lugar, intentaremos ver qué usos pueden ser considerados modernos, habiéndose perdido la palabra antigua, y cuáles pertenecen a un estadio primitivo del indoeuropeo. El método a seguir será hacer un estudio comparativo de todas las raíces que han dado lugar a estas designaciones y de las distintas alteraciones semánticas que han podido tener lugar. El estudio etimológico de la raíz en cuestión nos interesa sólo en la medida en que nos permita asociar la denominación de una parte concreta del cuerpo con una raíz más amplia, documentada a veces en otros campos semánticos. No se trata, pues, de hacer un profundo análisis etimológico, sino de ver de qué manera se han producido en las lenguas históricas las distintas designaciones.

\section{Designaciones de 'Mano'}

La palabra 'mano', o mejor dicho, la forma que sirve para designar esta parte del cuerpo, no está representada de manera unívoca en todas las lenguas. Tradicionalmente se ha considerado que la forma más

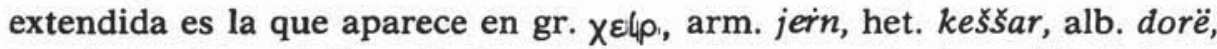
toc. A tsar, B șar ${ }^{2}$. A partir de estas formas se reconstruía un ide. " ghersprocedente de "gher- 'agarrar', raíz que se encuentra bien documentada en varias lenguas (cf. ai. hárati 'tomar consigo, llevar', haras 'poder', etcétera) ${ }^{3}$. Sin embargo, han surgido problemas que ponen en tela de juicio, por un lado, la derivación a partir de esta raíz, y por otro, la equiparación fonética de las formas anteriormente citadas. Hagamos un breve estado de la cuestión.

En primer lugar, la forma hetita y tocaria no permiten reconstruir * ghers- a no ser que se postule que ha habido una metátesis de $-r s>$ -sr, posible para algunos ${ }^{4}$. Esto pone en duda el parentesco con la raíz "gher- 'agarrar'. Las demás lenguas no ayudan demasiado a la resolución del problema ya que griego, armenio y albanés tienen el mismo resultado final: $-r$ tanto si se trata del grupo $-r s$ como $-s r$. En griego, la reconstrucción *ghers- explicaría la forma eolia $\chi \eta \rho \rho-$, dór. $\chi \eta \rho$, át. $\chi \varepsilon l \rho$. Chantraine parte de ${ }^{*}$ gher- que con la $-s$ del relacionador habría dado

2 Cf. a este respecto C. D. Buck, A Dictionary of Selected Synonims in the principal IE Languages, Chicago y Londres, $1965_{2}$, p. 238 ss.

3 J. Pokorny, Indogermanisches Etymologisches Wörterbuch (IEW), I, Berna 1948-1959, pp. 442 y 447.

4 Cf. J. Duchesne-Guillemin, «Hittite kèš̌ar 'main', indo-iranien žasta- et leur correspondants», BSL 39, 1938, pp. 211-221; W. Belardi, RSO 23, 1948, p. 694 ss. 


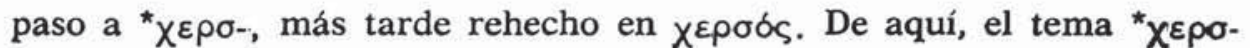
habría sido llevado a todo el paradigma ${ }^{5}$. La existencia de compuestos

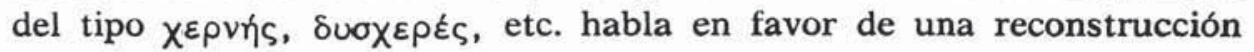
*ghers- para el griego.

El armenio jern procede de *ghers-ṇ, aunque también sería posible *ghesr $n$ n con el mismo resultado. La $n$ como es sabido procede de la desinencia de acusativo $-m$, habida cuenta de la confusión de Nom. y Acus. que ha tenido lugar en armenio ${ }^{6}$.

El hetita presenta kešsar, neutro y keššeraš de género común y tematizada a partir del neutro ${ }^{7}$. La reconstrucción *ghesr- Laroche la explica admitiendo la posibilidad de una metátesis de $-r s$ a $-s r$, lo cual permite asociar la palabra con la raíz ${ }^{*}$ gher- ${ }^{8}$. El resto de las formas más arriba mencionadas como pertenecientes a esta raíz presentan dudas fonéticas más serias. Así, el alb. dorë que procede de *ghērā con pérdida de $-s^{-9}$ y cuyo vocalismo largo no ha sido explicado satisfactoriamente. Ello hace que se haya comparado con formas pertenecientes a otras raíces, como gr. $\delta \omega \hat{p} o$ 'palma de la mano', let. dùre 'puño', a. irl. dorn 'puño' 10 .

Igualmente dudosa es la forma latina $h \bar{i} r$, ir documentada una sola vez en un pasaje oscuro de Lucilio, y que podría tratarse de una forma ficticia puesta de relieve por los gramáticos posteriores ${ }^{11}$. Su relación

5 H. Frisk, Griechisches Etymologisches Wörterbuch, Heidelberg 195472, s. u. XElp; P. Chantraine, Morfologia Histórica del Griego, Madrid 1974, p. 53 y Dictionnaire étymologique de la langue Grecque, París 1968-1980. En dórico está atestiguado Xepऽ (Timocreonte de Rodas, 9, ed. Bergk).

6 Cf. A. Meillet, Esquisse d'une Grammaire Comparée de l'armenien classique, Viena $1936_{2}$, p. 83; H. Hübschmann, Armenische Grammatik, Hildesheim-Nueva York 1972, I, p. 470. Specht lo deriva de ghérsēn 'agarrador', cf. Der Ursprung der Indogermanischen Deklination, Gotinga 1944, p. 75.

7 Cf. H. Kronasser, Etymologie der Hethitischen Sprache, Wiesbaden 1962-66, p. 275 y E. Sturtevant, «The parts of the body in Hittite», Language 4, 1928, p. 124; J. Tischler, Hethitisches Etymologisches Glossar, Innsbruck 1977, 3, pp. 560-562.

8 E. Laroche, Dictionnaire de la Langue Louvite, París 1959, p. 139 ss., «Etudes lexicales et étymologiques sur le hittite», BSL 58, 1963, p. 79, «Études de Linguistique anatolienne, 3. Le nom de la main», $R H A$ 76, 1963, p. 79. En luvita aparece išsari $=$ keššar con pérdida de la consonante inicial. Cf. también A. J. J. Van Windekens, AION-L 4, 1962, p. 24 ss. y M. Scheller, «Der angebliche Lautwandelkeilinschriften ki-> luw-i», IF 69,1964 , pp. 38-41.

9 Cf. G. Meyer, Etymologisches Wörterbuch der albanesischen Sprachen, Estrasburgo 1981, s. u.; C. Haebler en Sprache 13, 1967, p. 83 lo pone en duda, y lo mismo J. Schindler en IF 72, 1967, p. 244 ss.

10 Cf. F. Bechtel, Lexilogus zu Homer, Hildesheim 1964, p. 106 y W. Winter, «Die Vertretung indogermanischer Dentale in Tocharischen», IF 67, 1962, p. 28.

11 Lucilio en Cic. Fin. II 8, 23 (cf. ap. crít.). En la mayoría de las ediciones no aparece, y a nuestro juicio se trata de un error de interpretación a pesar de Vaniðek, Etymologisches Wörterbuch der Lateinischen Sprache, Leipzig 1881, p. 93 
con "gher- ha sido puesta en duda por Solmsen ${ }^{12}$, si bien podría tratarse de un préstamo del osco al latín emparentado con $[h]$ errins 'caperent' como ha mostrado Duchesne-Guillemin ${ }^{13}$, quien explica, además, el paso de $-s r$ a $-r$ en latín de la misma manera que ide. *uesr $>$ lat. uēr. El vocalismo largo $-\bar{i}<{ }^{*}-\bar{e}$ estaría debido a su procedencia del osco.

Las formas tocarias han planteado discusiones etimológicas que hacen alusion, por una parte, a la procedencia del grupo $t s$ - en tocario, y por otra, a la correspondencia entre toc. A tsar y B $\operatorname{sar}^{14}$. Generalmente, se admitía la procedencia del grupo $t s$ - de dentales y guturales palatalizadas, por lo que su relación con gr. $\chi \varepsilon i \rho$, arm. jernn, het. keššar, etcétera estaba asegurada ${ }^{15}$. Sin embargo, las dificultades de orden fonético obligan a replantear otras hipótesis como la de Bonfante ${ }^{16}$, quien desde el principio partió de la raiz *dher- 'sostener', bien documentada en indoeuropeo. Otros como Schneider parten de una raíz *der- 'desgarrar' que presenta dificultades fonéticas y Winter lo aproxima a gr. $\delta \omega \hat{p o v}$ y alb. dorë ${ }^{17}$. Ultimamente Van Windekens, quien desde el principio había defendido la procedencia de $t s$ - de dentales y guturales palatalizadas, por un lado, y la identidad de toc. A tsar B șar, ha pasado a derivar toc. A tsar de ide. *dheros 'la que sostiene', mientras que B șar lo deriva de ide. *ser- 'preservar' (cf. avést. haraiti 'proteger, cuidar', lat. seruare, etc.), con lo que B șar significa propiamente 'la que preserva' ${ }^{18}$. Todo ello trae como consecuencia que sólo exista una equivalencia segura entre las formas griegas, armenias y hetitas, dado que el alb. dorë también presenta dificultades. Estas lenguas designarían la mano con un derivado de la raíz *gher- 'agarrar' que, como luego veremos, no tiene nada de extraño.

En este apartado hemos de referirnos también a un grupo de formas cuya vinculación con las anteriores es objeto de controversia. Se

y de Meillet, Introduction a l'étude comparative des Langues Indo-Européennes, reimp. 8.8 ed., Alabama 1964, p. 407.

12 Beiträge zur Geschichte der Lateinischen Sprache», KZ 34, 1897/98, p. 3.

13 A. c., p. 213

14 Cf. Schindler, a. c., p. 244; Van Windekens, Le Muséon 64, 1951, p. 209, «Êtudes de Grammaire historique et comparée du Tokharien», AION-L 4, 1962, p. 24; H. Pedersen, Tocharisch vom Gesichtspunkt der Indoeuropäischen Sprachvergleichung, Copenhague 1941, p. 236.

15 Cf. A. Meillet, BSL 32, 1931, p. 60; Van Windekens, «Etudes de Phonétique tokharienne VI», Orbis 13, 1964, pp. 220-231; Duchesne-Guillemin, a. c., p. 211 ss.

16 En Mélanges Bally, Ginebra 1939, p. 20 ss., y recientemente en BSL 75, 1980 , p. 183.

$17 \mathrm{~K}$. Schneider, «Zu einigen Benennungen von Teilen des menschlichen Körpers im Tocharischen*, IF 58, 1941, p. 173 ss.; W. Winter, a. c., p. 28.

18 Le Tokharien confronté avec les autres langues indo-européennes, Lovaina 1975, I, p. 521. 
trata de ai. hasta, av. zasta 'mano' 19: La reconstrucción tradicional partía de un ide. *ghesto-, lo cual es imposible de mantener si tenemos en cuenta la proximidad de estas formas con lit. pažastìs, pažastè 'espacio bajo el brazo, axila' ${ }^{20}$. Las formas lituanas excluyen la -e- de la rafz originaria; habría, pues, que reconstruir *gho-/-ast- atendiendo al resultado en indoiranio y lituano. También se ha querido relacionar con lat. prēsto, de etimología dudosa y discutida ${ }^{21}$.

Finalmente hay que mencionar el término homérico đoyootós 'brazo'

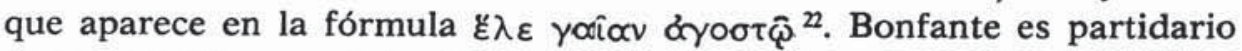
de su relación con ai. hasta, av. zasta y reconstruye " ${ }^{*}$ hosto ${ }^{23}$, mientras que Pokorny incluye óyootós en la raíz "ger-, gere- 'recoger, reunir', a la que pertenecen igualmente let. gùrste < gursti 'puño' 24. Cowgill propuso una reconstrucción ${ }^{*} H g o s t$ - que vendría a explicar tanto la aspiración del indio hasta, como la $\propto$ de $\alpha^{\prime} \gamma o \sigma \tau \delta \varsigma^{25}$, pero que pone en duda su relación con *ghers-, que es bastante verosímil. Las formas citadas anteriormente se encuadran en un marco más amplio de partes del cuerpo provistas del sufijo -st- como por ej. ai. gabhasti 'antebrazo',

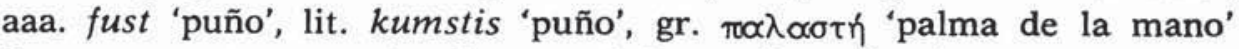
frente a $\pi \propto \lambda \measuredangle \mu \eta$, etc. Las formas ai. hasta, lit. pažastè, etc. suponen en cualquier caso un desarrollo específico dentro de un marco dialectal muy concreto.

Otro bloque de designaciones para la mano lo constituye el grupo formado por lat. manus 'mano', umb. manuve 'in manu' (tema en - $u$ ), osc. manim 'manum' (tema en -i), manf (ac. pl.), a. isl. mund 'mano' (mṇtós), a. ing. mund, aaa. munt 'mano, tutela' ${ }^{26}$. El tema sería el mismo que para lat. mancus, manceps, mandō y a. isl. mundr, formas todas ellas

19 Cf. Duchesne-Guillemin, a. c., pp. 211-221; M. Mayrhofer, Kurzgefasstes Etymologisches Wörterbuch des Altindischen, Heidelberg 1958, p. 588.

20 R. Trautmann, Baltisch-Slavisches Wörterbuch, Gotinga 1923, p. 367; J. Endzelin, Comparative Phonology and Morphology of the Baltic Languages, La Haya 1971, p. 122.

21 Cf. J. Wackernagel, Versammlung der Schweizerische Philologenverbands, 1919. p. 168 que lo deriva de prai-hestō y lo relaciona con hasta- 'mano'. A Ernout y Meillet les parece sugestiva tal hipótesis, cf. Dictionnaire Etymologique de la Langue Latine, París $1967_{14}$, s. u. presto; A. Walde-J. Hoffmann, Lateinisches Etymologisches Wörterbuch, Heidelberg 1938, II, p. 356.

22 Cf. Wackernagel, Altindische Grammatik I, Gotinga 1896, reimp. 1957, p. 249.

23 Cf. I Dialetti Indeuropei, Brescia 1976, p. 88; también M. Morani, Aevum 54, 1980 , p. 104.

24 Pokorny, IEW I, p. 447.

25 Cf. W. Cowgill en Evidence for Laryngeals, ed. W. Winter, La Haya 1965, p. 152, y reseña de Hammerich en Lingua 22, 1969, p. 203.

26 Pokorny, IEW I, p. 740. 
con un amplio sentido jurídico tanto en latín como en germánico ${ }^{2}$, derivado seguramente del uso de 'mano' como símbolo de fuerza y de la autoridad marital del hombre sobre la mujer.

El término, a primera vista, se halla restringido a un área dialectal concreta, a saber, la italo-germánica. Por otra parte, no se ha establecido hasta ahora la vinculación con alguna raíz indoeuropea de más amplia difusión y las formas atestiguadas presentan diferencias morfológicas: temas en vocal en las lenguas itálicas, y en consonante en germánico. No es de extrañar, por tanto, que la opinión más generalizada sea la de que nos hallamos ante palabras aisladas de origen oscuro y quizá ritual ${ }^{28}$. Es posible, sin embargo, que se trate de un derivado de la raíz ${ }^{*} m H-(r) /(n)$ con el sentido de 'tocar, palpar'. A dicha raíz pertenece, por ejemplo, el verbo griego $\mu \propto l o \mu \propto$ cuyo parentesco etimológico fue visto ya por Belardi ${ }^{29}$. Este verbo presenta dos significados: por una parte 'palpar, tocar' y por otra 'ambicionar, perseguir algo'. En el primer caso rige acusativo y en el segundo, genitivo. Tanto Belardi como Bechtel ${ }^{30}$ son partidarios de ver dos verbos distintos, uno procedente de ${ }^{*} \mu \alpha$ o $\mu_{\alpha} \propto$ y otro de ${ }^{*} \mu \alpha \sigma i o \mu \alpha t$.

Esta explicación es innecesaria. En Homero aparecen mezclados los usos con un sentido y otro, y el significado 'ambicionar' se encuentra la mayoría de las veces unido al tema de presente. De esto se deduce que este sentido puede ser un desarrollo del valor conativo o, simplemente, desiderativo del presente, sin que sea preciso postular dos verbos distintos y de distinto origen. Hay que partir, por lo tanto, de una raíz indoeuropea * $m \bar{a}$ 'tocar, palpar' que también está presente en het. maniyab 'entregar en mano, transmitir' y 'administrar' ${ }^{31}$. Este último significado es posterior a los otros y probablemente se trata de un calco del acadio ${ }^{32}$.

Pokorny recoge una raíz *ma- 'hacer señas con la mano' 33 , y que podría estar igualmente relacionada con māa. Derivados de ella son gr. $\mu \eta v u ́ \omega$, a. esl. namajo 'hacer señas con la mano', lit. moju ${ }^{34}$, etc.

\footnotetext{
27 Ernout-Meillet, Dictionnaire..., p. 386 y J. de Vries, Altnordisches Etymologisches Wörterbuch, Leiden $1962_{2}$, p. 395.

2 Cf. G. Devoto, Origini Indeuropee, Florencia 1962, p. 264.

29 Maia 2, 1949, p. 277 s.

30 O. c., s. u. $\mu \alpha l o \mu \alpha$ l.

31 Cf. J. Friedrich, Hethitisches Wörterbuch, Heidelberg 1952, p. 135; H. Kronasser, o. c., p. 432 ; la relación ya había sido vista por Ch. L. Mudge, «Ten hittite Etymologies», Language 7, 1931, p. 252.

32 A. Götze, Hattušiliš, Leipzig 1925 , p. 60 ss.; Kronasser, o. c., p. 432; Van Windekens incluye en esta raíz toc. B maçce 'puño', ac. sg. macc, de ide. "mn-stẻ(n), cf. Le Tokharien confronté... I, p. 281.

33 IEW I, p. 693.

34 Cf. R. Trautmann, Balt. Slav. Wört., p. 166.
} 
Se ha comparado también con lat. manus la palabra griega $\mu$ óxp 'mano' que aparece en Píndaro ${ }^{35}$. Forssman lo interpreta como un falso corte a partir de eujuopín ${ }^{36}$. Meillet, por su parte, derivaba esta palabra de una raíz *mer- a la que pertenecería también ai. mrśati 'tocar' con alargamiento velar ${ }^{37}$ y que se puede relacionar a su vez con toc. A

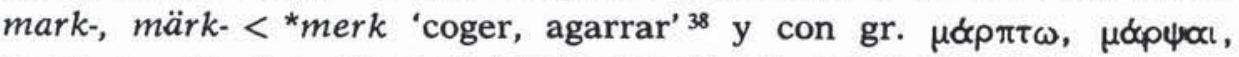
$\beta p \nless \zeta \propto c^{\prime} \sigma u \lambda \lambda \propto \beta \varepsilon i ̂ v$ glosa de Hesiquio; cf. alb. marr < marnō 'sostener, coger'. A la vista de estos datos, hemos de pensar en la existencia de una raíz ${ }^{*} \mathrm{meH}_{2}$ quizá con el significado 'tocar, palpar' que recibió diferentes alargamientos, y en algunas lenguas se especializó en la designación de la mano, concretamente en itálico y germánico, donde no existe la palabra derivada de *ghers. Esto hace pensar en una distribución dialectal de las dos raíces.

Reducida al área germánica, existe otra forma de designar la mano: a. ing. hand, aaa. hant, a. nórd. hond, gót. handus ${ }^{39}$. La etimología no es segura y se supone, generalmente, que procede de una raíz ${ }^{*} k e m-t$ / $k m-t$. El parentesco más plausible es con el verbo gót. fra-hinpan, compuesto que significa 'agarrar, perseguir', y con el a. ing. huntian 'cazar' 10 . También se ha puesto en relación con gr. kœtớ, irl. cēt, het. katta ${ }^{41}$, lo cual es bastante inverosímil, dado el carácter adverbial de estas palabras. Está muy generalizada la opinión que consiste en derivar de *kem-t/km-t el numeral 10: lat. decem, gr. $\delta \varepsilon \kappa \alpha$, ai. daśa, etc. a partir de *de-kmtom < due-kmtom que significaría originariamente 'dos manos' ${ }^{42}$. Sturtevant piensa que el segundo miembro de *de-kmt puede ser conectado con gót. handus, siendo un tema en consonante en ide. que fue transferido a los temas en $-u$ en germánico ${ }^{43}$. Por el contrario, Kluge cree que el significado originario de *kont habría sido ' 10 ' y de

35 Pi. Fr. 310 Snell-Maehler.

36 Untersuchungen zur Sprache Pindars, Wiesbaden 1966, p. 135 ss.

37 Cf. MSL 17, 1911/12, p. 62.

38 A. J. J. Van Windekens, Léxique étymologique des Dialectes Tokhariens, Lovaina 1941, p. 62.

39 Cf. A. Walde y J. Pokorny, Vergleichendes Wörterbuch der indogermanischen Sprachen, Berlín-Leipzig 1930, I, p. 460; S. Feist, Vergleichendes Wörterbuch der gotischen Sprachen, Leiden $1939_{3}$, p. 244.

40 J. Grimm, Deutsche Grammatik, Gotinga 1819, II, p. 35, pensaba que era un ablativo de hinpan 'cogedor'. Meillet rechaza esta relación, cf. BSL 29, 1929, pp. 86, 36.

41 Cf. M. Blankenstein, «Griechische $k \alpha \tau \alpha$ und seine Verwandten», IF 21, 1907 , p. 109.

42 Cf. S. Bugge, «Etymologische Beiträge», $B B$ 14, 1889, p. 72; ha sido admitida también por 0 . Szemerényi, Studies in the Indo-European Numerals, Heidelberg 1960, p. 69.

43 «Hittite KATTA(N) and Related Words», AJPh 48, 1927, pp. 247-257. 
aquí thabría pasado a equivaler a 'dos manos', a partir de lo cual se habría especializado su uso para 'mano' 44.

La principal objeción a esta hipótesis es que nos hallamos ante una palabra documentada sólo en germánico y no existen testimonios de otras lenguas donde el numeral ' 10 ' se haya desarrollado a partir de una raíz significando 'mano' ${ }^{4}$. Respecto al término gót. handus, aaa. hant, etc. se han querido poner en relación con arm. kant 'puñado' sin que resulte aceptable tal comparación 4 .

Lo más probable es que se trate de una formación reducida al área germánica, de etimología no segura, procedente quizá de una raíz con el significado de 'agarrar', de donde derivan tanto la palabra para 'mano', como el verbo gót. fra-hinpan, cuyo parentesco semántico es indudable. Resulta a todas luces sintomático el hecho de que Pokorny no incluya esta raíz entre las indoeuropeas.

Las lenguas bálticas y eslavas tienen una forma propia para desig. nar la mano: lit. rankà, let. ruoka, a. esl. rqka > *rankā 'mano', ide. *uronka ". La etimología habría que conectarla con la raíz *uerk- 'girar, torcer', con infijo nasal *ure-n-k, de donde proceden también germ. *uranhō: a. isl. vrá 'ángulo, esquina' 48 , lat. tardío branca 'pata' 49 *uranka (galo). Por otra parte, también se ha puesto en relación con el verbo lituano riñkti 'recoger', que probablemente procede de la misma raíz ${ }^{50}$. Se trata en cualquier caso de un término documentado con el significado de 'mano' en el área baltoeslava, siendo, por tanto, un desarrollo especifico de este grupo dialectal. No obstante, la raíz entronca con una serie de ellas que con el significado de 'torcer' han dado lugar a la palabra que designa el brazo en ciertas lenguas indoeuropeas.

Finalmente hemos de ocuparnos de la raíz ${ }^{*} p e l H_{z}-m-/ p l H_{\tau}$ de amplia documentación en indoeuropeo, y cuyo significado es 'plano'. De ella derivan gr. $\pi \lambda \propto \tau u ́ s$, lat. planus, etc. En algunas lenguas designa la 'palma de la mano': gr. $\pi \propto \lambda \propto \alpha \eta$, lat. palma, a. ing. folm, etc. En a. irl. ha tenido lugar un desplazamiento semántico, fácil de ver, y ha pasado a significar 'mano': $l \vec{a} m^{51}$. En a. indio existe pāní 'mano' procedente

44 Etymologisches Wörterbuch der Deutschen Sprache, Berlín-Leipzig 1930, p. 230.

45 Cf. a este respecto la objeción de Thurneysen en $K Z 26,1883$, p. 84.

4 Cf. J. Scheftelowitz, «Zur altarmenischen Lautgeschichte», BB 29, 1905, p. 26.

47 Cf. Pokorny, IEW I, p. 1155; R. Trautmann, Balt. Slav. Wört., p. 237.

4 Cf. J. J. Mikkola, «Zur slavischen Etymologie», IF 23, 1908, p. 120.

4 Cf. J. Corominas, Diccionario Crítico Etimológico de la Lengua Castellana, Madrid 1974, I, p. 509 , s. u. branza.

so Cf. F. Specht, o. c., p. 158; L. v. Patrubańý, «Lituanica», IF 32, 1913, p. 239; M. Vasmer, Russisches Etymologisches Wörterbuch, Heidelberg 1950-1958, p. 545.

51 V. Pokorny, IEW I, p. 806 y H. Pedersen, Vergleichende Grammatik der keltischen Sprachen, Gotinga 1913, II, p. 561. 
de *pārní, que, a pesar de haber sido emparentada con las formas anteriores por Meillet ${ }^{52}$, presenta dificultades de interpretación ${ }^{53}$.

Con esto queda vista lo referente a la designación de la mano en las lenguas indoeuropeas; hagamos ahora un resumen de lo que se ha dicho. En primer lugar, la noción de 'mano' en indoeuropeo se sirve para su expresión de ciertas raíces cuyo significado parece aludir, sin duda, a la actividad que dicha parte del cuerpo realiza. Así, tenemos derivados de *gher- 'coger, agarrar', *dher- 'mantener', *mā- 'tocar, palpar', *kem- 'agarrar'. Otras veces, deriva de una raíz que designa una parte de la mano y de ahí ha pasado a significar 'mano' por metonimia. Éste es el caso de pel-/plä- "liso, llano' que con sufijo $-m$ designaba la parte plana de la mano, es decir, la palma, y en a. irl. se utiliza sólo para 'mano'. En las lenguas bálticas y eslavas también ha tenido lugar un desplazamiento semántico ya que la raíz *uer-k- 'torcer' es la que ha originado la designación del brazo que sirve igualmente para 'mano'. En segundo lugar, todas estas raíces, a excepción de *kem-, están bien documentadas en las lenguas indoeuropeas dando origen a palabras de variada índole. Sólo *kem- se encuentra aislada dentro del grupo germánico. De ello se deduce la falta de unanimidad en la designación de 'mano' dentro del ámbito indoeuropeo. Cada lengua o grupo de lenguas ha optado por una solución dentro de un abanico de posibilidades. Esto viene favorecido por varios factores: $1 .^{\circ}$ ) La multiplicidad de funciones de la mano, que abarca desde su utilización como instrumento de trabajo, de caza, etc. $2^{\circ}$ ) Sus simbolismos en la antigüedad, de tipo jurídico en unos casos, rituales y mágicos en otros ${ }^{54}$, que la hacen susceptible de una gran inestabilidad lexical. Hay que hacer notar, sin embargo, que su concepción como instrumento destaca por encima de los otros usos. Sólo la palabra manus y sus correspondencias en las lenguas germánicas podría ponerse en relación con ciertos simbolismos jurídicos y mágicos de la mano.

Este estado de cosas hace que resulte imposible la reconstrucción de una sola forma panindoeuropea para 'mano' y en un estadio antiguo, menos aún. Todos los testimonios que poseemos corresponden a áreas concretas más o menos relacionadas dialectalmente. Quizá las formas procedentes de *ghers- cuyo testimonio encontramos en hetita kešsar hacen pensar en una mayor antigüedad de ellas. Sin embargo, el hecho de que no haya ninguna huella en germánico o eslavo debe

\footnotetext{
52 Cf. Introduction..., p. 408 y $M S L 20,1916-18$, p. 289 ss.

53 Véase a este respecto Mayrhofer, Kurzg. Etym. Wört., s. u., donde se trata como un pracritismo temprano quizá de origen Pamir.

\$4 Cf. G. Devoto, Origini Indeuropee, Florencia 1962, p. 214.
} 
ponerse en relación con que estas lenguas desarrollaron desde muy pronto otra palabra derivada de una raíz distinta. No estamos de acuerdo con Devoto en su afirmación de que las formas lat. manus, osc. manim, etc., por aludir a una concepción abstracta de la mano, han de ser más antiguas que las otras ${ }^{55}$. Más bien hay que pensar en un desarrollo paralelo, sin que se pueda establecer a ciencia cierta la cronología.

\section{Designaciones DE 'BRAZo'}

Lo mismo que ocurre con la mano, el brazo no tiene una designación única que abarque a todas las lenguas indoeuropeas. En primer lugar, existe un grupo de lengua que utiliza un derivado de la raíz ${ }^{*}(H) e r H^{i-}$ 'ajustar' ${ }^{56}$, la cual se utiliza más frecuentemente para designar el hombro. Así, ai. ìrmá- 'brazo' (C/C y alargamiento), avést. arama'brazo' (C/C a), lat. armus (P sin apoyo vocálico) 'omoplato, hombro', a. esl. ramo, rame (P) 'hombro', gót. arms (P) 'brazo', a. prus. irmo (C/C) 'brazo', arm. armukn 'codo'. La designación del brazo a partir de esta raíz se habría originado por la noción de 'junta' o 'articulación' y en principio se habría aplicado al hombro, incluso al codo, como lo muestran lat. armus, a. esl. ramo, rame, incluso a. i. irmá-, arm. ar$m u k n^{5}$. Frecuentemente se aplican los derivados de la raíz ${ }^{*}(H) e r H^{i-}$ a las patas de los animales, como sucede en latín, donde armus es utilizado para los animales, mientras que humerus se aplica en el hombre ${ }^{58}$. Lo mismo sucede en indio irmá- que se usa con animales y bāhu'brazo' en los humanos ${ }^{59}$. La raíz presenta problemas fonéticos que no interfieren, creemos, en lo que aquí estamos tratando ${ }^{60}$.

Tradicionalmente se han considerado formas idénticas gr. $\pi \eta \bar{x} \cup \varsigma$ y ai. bāhú, avést. bāzau- 'brazo'. A estas lenguas habría que añadir, con otros alargamientos, aaa. buog 'hombro', a. nórd. bōgr 'brazo' y 'hombro', y seguramente toc. A poke, B pauke 'brazo'. En los diccionarios etimológicos indoeuropeos no existe una raíz de la que sea posible

55 Cf. o. c., p. 264.

56 Cf. F. R. Adrados, Estudios sobre las Sonantes y Laringales Indoeuropeas, Madrid $1973_{2}$, p. 257.

5 Según Hübschmann, o. c., p. 425, armukn estaría influida en su formación por mukn 'ratón' y 'músculo', cf. a. esl. myšica 'brazo', lat. musculus 'ratoncillo' y más tarde 'músculo'.

58 Cf. Ernout-Meillet, Dictionnaire..., p. 74.

59 Cf. Mayrhofer, Kurzg. Etym. Wört., s. u.

60 Cf. E. P. Hamp, «Arm, Shoulder», JIES 10, 1982, pp. 187-189. 
derivar este grupo de formas. De ahí que generalmente se reconstruyera una palabra *bhāghús considerada como un vocablo primario. Sin embargo, hay que mencionar el excelente trabajo de Benveniste ${ }^{61}$ donde pone de manifiesto la existencia de una raíz bhägh- 'extender' en oseta ${ }^{62}$ que estaría en la base del iranio bāzu- 'brazo', designando el brazo como 'extendido'. Benveniste considera que la $-u$ de *bhäghús es sufijal y no pertenece a la raíz, basándose en la no existencia de tal $-u$ en toc. A poke.

La correspondencia entre gr. $\pi \hat{\eta} \chi \cup \varsigma$, ai. bāhu- con toc. A poke, B pauke ha sido objeto de controversia. Ya Pedersen ${ }^{63}$ y Schulze ${ }^{64}$ veían la dificultad que su etimología entrañaba. A pesar de ello, la opinión de los estudiosos es unánime en este sentido, y todos ellos, aun con dificultades, relacionan las formas indoiranias y tocarias ${ }^{65}$. El más ardiente defensor de esta postura ha sido Van Windekens, quien siguiendo una idea ya mencionada por Lane, pero propiamente de Sapir ${ }^{66}$, mantiene que la $-u$ - de pauke es consecuencia de haber influido la $-u$ de *bhāghus sobre la primera sílaba. Así, "bhāghus habría dado toc. ${ }^{*} p \bar{a} k u$ de donde ${ }^{*} p a^{u} k u$ - y luego desaparece de la sílaba final ${ }^{67}$. La idea, aun siendo sugestiva, ha sido rechazada por Bonfante totalmente ${ }^{68}$. Este autor se basa en que cuando en tocario B existe un diptongo, éste es siempre más antiguo que su monoptongación en el dialecto A. Por tanto, habría que partir de la forma toc. B pauke. Esta forma estaría entonces relacionada con la raíz *bheugh- 'doblar' que ha dado aaa. -bogo, a. ing. boga, ing. bow, etc. Estas formas germánicas aparecen frecuentemente como segundo término de compuestos que designan el codo, como luego veremos.

Así pues, se trataría en este caso de una sustitución en tocario del nombre del brazo por el del codo, cosa nada infrecuente si tenemos en cuenta que en ai. existe un derivado de la raíz *bheugh-con el significado de 'brazo': bhujá-69. Dado que los testimonios que existen en

61 «Analyse d'un vocable primaire: indo-européen *bhāghu- 'bras'», BSL 52, 1956, pp. $60-71$.

62 i-woez 'extender', iran. baz, cf. a. c., p. 64.

63 Tocharisch..., pp. 42 y 74.

64 Kleine Schriften, Gotinga 1966, p. 255, nota 1.

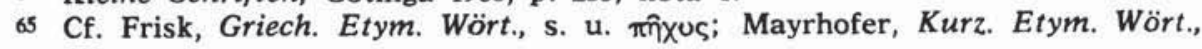
II, p. 429.

66 En G. S. Lane, Language 13-14, 1937/38, p. 35, nota 73.

67 Cf. A. J. J. van Windekens, Orbis 11, 1962, p. 191 y AION-L 3-4, 1961/62, p. 5.

68 \% Trois noms de parties du corps en Tokharien (A et B)w, BSL 75, 1980 , p. 186 ss.

69 Piénsese, por ejemplo, en gr. $\kappa \propto \mu \pi \hat{y}$ 'curvatura' que ha dado lugar a fr. jambe, it. gamba 'pierna'. 
tocario para avalar una $u$ otra teoría son escasos y de igual peso, nos resulta difícil inclinarnos por una de ellas. Ahora bien, la teoría de Bonfante no deja de ser sugestiva en dos aspectos: $1^{\circ}$ ) explica con verosimilitud la correspondencia entre los dialectos A y B y su pertenencia a la raiz *bheugh-, correcta desde un punto de vista fonético; $\left.2 .^{\circ}\right)$ restringe la forma *bhāghús a griego e indoiranio lenguas que han estado, evidentemente, relacionadas. Esto llevaría a pensar en un desarrollo relativamente reciente. Por otro lado, nos ofrece la correspondencia de formas tocarias y germánicas, que lejos de ser considerado un caso de préstamo, habría que verlo bien como un arcaísmo conservado en ambas lenguas, no relacionadas dialectalmente, bien como una tendencia que ya existía en indoeuropeo de derivar el nombre del brazo, codo, etc. de raíces que significaban 'torcer', 'doblar', etc. ${ }^{7}$.

En lo que respecta a su significado, hay que hacer notar que en griego $\pi \hat{\chi} \chi u \varsigma$ designa el brazo en oposición al antebrazo ${ }^{71}$ ( $\left.\beta \rho \propto \chi l \omega v\right)$, mientras que en las lenguas germánicas, a. nórd. bōgr, aaa. buog se aplican generalmente a las patas de los animales ${ }^{72}$.

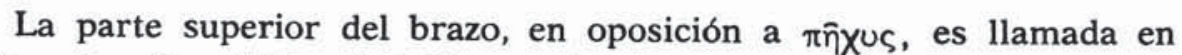

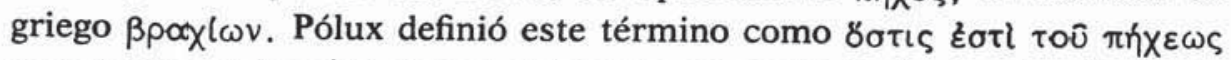

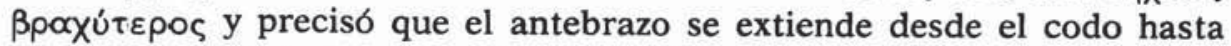
el extremo del dedo medio ${ }^{73}$. Etimológicamente $\beta \rho \alpha \chi l \omega \nu$ está derivado de *mregh-/mrgh-, es decir, la misma raíz de ßpoxús 'corto', y según Pólux se podría traducir por 'el más corto' ${ }^{74}$. Merlingen ha puesto en duda esta etimología, y lo relaciona con *uerg- 'trabajar', sin que haya una razón evidente para ello ${ }^{75}$. El latín brac(c)hium que aparece aplicado al brazo y también a la extremidad delantera de un animal (pata, pinza, etc.) se trata de un préstamo del griego ${ }^{76}$. En celta aparece gal. braich > bracchium, bret. brec' $h$, irl. brach 'brazo', que está a su vez tomado del latín. Según Van Windekens, la palabra tocaria B märkwac

ᄁ Véanse en este sentido las formas derivadas de *uerk- 'torcer' en eslavo y en báltico. Sería sugestivo el conjugar las dos teorías a base de postular una laringal ${ }^{\star} H^{u}$ en la raíz que habría dado lugar a $\pi \hat{x}$ ux y *bheugh-, pero los datos que aportan las lenguas históricas parecen descartar esta posibilidad.

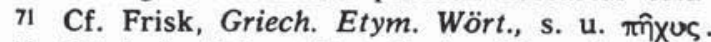

72 Cf. J. de Vries, Altnordisches Etymologisches Wörterbuch, Leiden 1962 , p. 47.

73 Pólux II 138, 158.

74 La etimología está explicada claramente por Ruijgh, «Les noms en -won(-äwon, -iwon), -uon en grec alphabétique et en mycenien», Minos NS 9, 1968, p. 147.

75 Cf. W. Merlingen, Eine ältere Lehnwörterschicht im Griechischen II, Viena 1967, p. 6.

76 Cf. Ernout-Meillet, Dictionnaire..., p. 75. 
'muslo', estaría igualmente relacionada con la raíz que nos ocupa, siendo $-a c$ un alargamiento secundario ${ }^{n}$.

Se puede deducir de lo visto hasta ahora que la noción de brazo deriva en unos casos del desplazamiento semántico de la noción de 'ajustar' aplicada, generalmente, al hombro o al codo, y en otros de la de 'extender' o 'doblar' que harían alusión a las funciones del brazo. Las lenguas han elegido entre las diferentes posibilidades, siendo la más extendida la forma derivada de ${ }^{*}(H) e r H^{i}$. De nuevo nos encontramos con una serie de tendencias entre las que escogen las diferentes lenguas, sin que sea posible reconstruir un solo término para designar el brazo. Por un lado, griego, indoiranio y germánico utilizan un término derivado quizá de *bhagh- 'extender', pero estas mismas lenguas presentan igualmente derivados de ${ }^{*}(H) e r H^{i}$ para expresar, en unos casos, el miembro de un animal, o bien nociones de 'junta' o 'articulación' como en el caso del griego. La forma $\beta p \propto x\lfloor\omega v$ es exclusiva del griego, de donde ha pasado al latín y al celta. Por otro lado, hay que tener en cuenta que muchas lenguas utilizan la misma palabra para 'mano' y 'brazo', considerando que ambas partes forman un conjunto único. Así, irl. läm, lit. rankà, a. nórd. họnd. En griego moderno $\chi \underline{\varepsilon} \rho \mathrm{l}$ es la palabra usada popularmente tanto para 'mano' como para 'brazo'.

\section{Designaciones DE 'CODo'}

La raíz más frecuente de la que deriva el codo en las lenguas indoeuropeas es ${ }^{*}\left(\mathrm{H}_{3}\right) \mathrm{leH}^{\mathrm{i}{ }^{-}}$'doblar', 'torcer', aunque es bastante habitual el intercambio de formas para designar el codo y el antebrazo. Así, en gr. $\omega \lambda \varepsilon \varepsilon \nu \eta$ 'codo' y 'antebrazo', $\omega \lambda \epsilon \kappa \kappa \rho \bar{\alpha} \nu o \nu$ 'codo', compuesta de $\omega \lambda \varepsilon \nu \delta \delta$ -

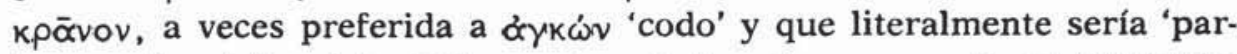

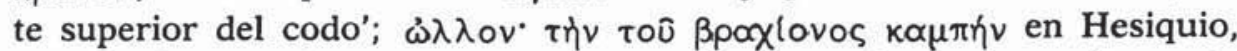

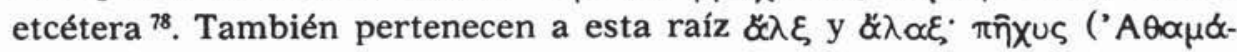
$v \omega \nu)$, Hsch.

En latín aparece ulna $>$ *olina $\left(H_{3} l-H^{i} \rho^{o}\right)$ 'antebrazo' y 'codo', que se usa, generalmente, como medida ${ }^{79}$; en a. irl. uilenn 'ángulo' ha dado

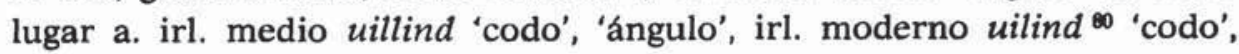

$\pi$ «Etudes de Phonétique tokharienne XIII», Orbis 19, 1970, p. 113 y «Sur l'origine indo-européenne de quelques mots tokhariennes IIn, Orbis 14, 1965, p. 502.

78 Cf. Pokorny, IEW I, p. 307.

79 Cf. Ernout-Meillet, Dictionnaire..., p. 1120.

so Sobre el paso de $\ln >$ el v. Pedersen, Keltische Grammatik II, p. 59. 
todas ellas procedentes de ${ }^{*} o l i ̄ n a{ }^{81}\left(H_{3 l} l-H^{\mathrm{i}}{ }^{o}\right)$. En ai. aratni-, avést. arəOna 'codo' $\mathrm{H}_{3} l \mathrm{lH}_{1^{-}}>$aratna-(i). Según Benveniste ${ }^{82}$ se trataría de un derivado feminizado de una raíz ampliada con el sufijo ${ }^{*}$-ter/-ten ${ }^{8}$. De esta misma raíz procede también āníh 'muslo' (ärni $<{ }^{\star} \bar{e} \ln i<{ }^{*} \bar{o} \ln i<$ $\left.{ }^{\star} H_{3} l-\right)$. Compárense también ai. arālaṇ 'curvo, corvo'. En albanés, existe lërë, pl. lans literalmente 'brazo desde el codo a la mano' ${ }^{84}$, es decir, 'antebrazo', procedente de * $(H)$ len $_{r}$ - . En armenio oln 'vértebra dorsal, hombro', a partir de ${ }^{\star} H_{3} l e H_{1}-n$, está igualmente emparentado.

Ampliado con el sufijo velar $-k$ - aparece en lit. úolektis 85 'cúbito' < *H$H_{3}$-ek-, alkúné, elkúné, a. prus. alkunis 'codo', y sin el sufijo en a. prus. woaltis, woltis 'cúbito, antebrazo'. En las lenguas eslavas está documentado lakztb 'codo' en búlgaro antiguo ${ }^{86}$. En germánico, la raíz está bien atestiguada: gót. aleina 'codo, cúbito' ( $<{ }^{*}$ olīna), ags. eln ( ${ }^{*}$ olǐna), aaa. elina, a. isl. $\ln ^{87}$. También aparece en composición con la raíz *bheugh'doblar': a. nórd. plnbogi, a. ing. elnboga (> elbow en ing. mod.), aaa. el(l)inbogo 'codo'. La recaracterización de la forma supone la pérdida del significado 'doblar' en la primera parte del compuesto.

Hasta ahora hemos visto los derivados de la raíz $\left(H_{3}\right) l e H_{t^{-}}$que significan 'codo', pero existen infinidad de formas relacionadas de un modo $\mathrm{u}$ otro con ella, y que hacen patente la presencia de tal raíz en todo el ámbito indoeuropeo. Así, toc. A äle, B alyiye 'palma de la mano' procedente de *olios < ${ }^{*} H_{3}$ l-ios $\$$; het. haliya 'arrodillarse' verbo temático con un causativo balinu 'hacer arrodillarse', con reduplicación y síncopa, balibla 'postrarse, ser sumiso'. Tanto la formación de la palabra como su significado y relación con 'rodilla', avalan la pertenencia a esta raíz ${ }^{89}$. También se encuentra en balbaltumari 'ángulo, esquina', bali 'cercado', hališšiya 'orlar, engarzar', etc. De todo lo visto hasta ahora se deduce que nos hallamos ante una raíz ampliamente documentada en

\footnotetext{
81 Cf. Feist, Vergl. Wört, der Gotischen Sprache, p. 35.

82 Origines de la formations des noms en Indo-Européen, París 1935, p. 105.

83 Cf. O. Szemerényi, «Iranica II», Die Sprache 12, 1966, p. 199.

84 Cf. G. Meyer, Etymilogisches Wörterbuch der albanesischen Sprache, Estrasburgo 1891, p. 233.

85 Cf. J. Endzelin, o. c., p. 33.

86 Cf. F. Miklosich, Etymologisches Wörterbuch der slavischen Sprachen, Viena 1886, s. u. lakzt b y R. Trautmann, Balt. Slav. Wört.

87 S. Feist, Vergleichende Wörterbuch der gotischen Sprache, Leiden 1939 3 , p. 35.

88 Así W. Krause, «Zur Frage nach dem nichtindogermanischen Substrat des Tocharischen*, KZ 69, 1949, p. 203.

89 La relación fue vista ya por Bernabé en Hetita y Laringales Indoeuropeas, tesis doctoral inéd., Madrid 1973, p. 91 ss. y «Raíces con dos laringales en Griego», RSEL 2, 1975, p. 380 . Pedersen relaciona het. baliya con lit. kẻlias 'rodilla', cf. Hittitisch und die andere indoeuropäischen Sprachen, Copenhague 1938, pp. 176-179; en contra A. Martinet, PICL 8, 1958, p. 51 y Phonetica 1, p. 22.
} 
indoeuropeo, si bien sólo en algunas lenguas se ha especializado en designar el codo. Por otro lado, la relación semántica entre el significado de la raíz ${ }^{*} H_{3} l e H_{T^{-}}$'doblar, torcer' y la palabra 'codo' es evidente.

En latín encontramos la palabra cubitus 'codo' que ha proporcionado la forma de designar el codo en la mayoría de las lenguas románicas (esp. codo, fr. coude, it. gomito, etc.). Su etimología no está unánimemente aceptada. Ya desde la antigüedad se ponía en relación con el verbo latino cubāre 'reclinarse' o, más bien, 'acodarse' ${ }^{90}$. Sin embargo, a pesar de haber sido la opinión más generalizada ${ }^{91}$, sigue sin haber un acuerdo unánime.

Pisani cree que se trata de un término emparentado con gr. kúßıtov 'codo' de origen sículo y con ai. gábhasti- 'brazo, mano', siendo una palabra de procedencia indo-mediterránea ${ }^{92}$. A su vez, lo pone en relación con het. gipešsarar 'codo' utilizado como medida. Rechaza categóricamente la relación con cubāre, admitida por Bonfante. Sin embargo, la identificación de cubitus con ai. gábhasti- y con het. gipešsar no está clara. La forma ai. gábhasti- deriva de la raíz *ghebh-/ghabh- 'coger, tomar' de donde proceden igualmente lat. habeo, a. irl. gaibid, etc. ${ }^{93}$ y se trata de una formación con sufijo -st- muy frecuente en formas secundarias de partes del cuerpo (cf. ai. hasta- 'mano', gr. $\pi \propto \lambda \propto \sigma \tau \gamma^{\prime}$ 'palma de la mano', lit. kumstis 'puño', etc.). La forma hetita gipešsar derivaría también de esta raíz. Pero para lat. cubitus, gr. kúßıtov hemos de buscar otra etimología más aceptable. En opinión de Frisk ${ }^{94}$, la forma griega deriva del latín, siendo ésta de origen sículo; por el contrario, Ernout y Meillet admiten la posibilidad de que ambas tengan origen suditálico ${ }^{95}$. Pisani cree que se trata de un préstamo del griego $\%$.

La etimología más acertada, a nuestro modo de ver, es precisamente la de Pokorny, quien deriva cubitus de la raiz *keu-/keua- 'doblar', 'torcer' y que puede recibir diversos alargamientos en labial, velar, etc. A ella pertenecerían también gót. hups, ags. hype, aaa. huf 'cadera'. Todos sus derivados hacen alusión a una articulación, por lo que no es extraño que aparezca entre ellos el codo. En griego, junto a kúßıтov

90 También G. Bonfante, Ricerce Linguistice 1, 1950, p. 258 propone esta relación.

91 Cf. Ernout-Meillet, Dictionnaire..., p. 237, Walde-Pokorny I, p. 374, WaldeHoffmann I, p. 297.

92 Paideia 8,1953 , p. 308 y 19, 1964, p. 286. Por otra parte, en $R S O 14$, p. 86 cree que ai. gábhasti- es una contaminación entre "gabha- (av. gav-, gava- 'mano') y ai. hasta.

93 Etimología de Cop, Schreibung und Lautung 1, 1964, p. 42.

94 Griech. Etym. Wört., s. u. кúßıтov.

95 Dictionnaire..., p. 153.

\% Cf. Le Lingue dell'Italia antica oltre il Latino, Turín 1964, p. 288 ss. 
aparece кúßos 'vértebra del cuello' 97, 'cavidad ilíaca del ganado' 98 y

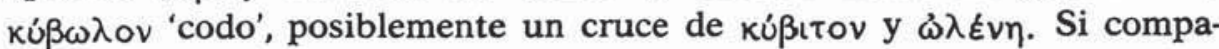
ramos con otras formas de designar el codo, vemos que todas ellas proceden de una raíz que significa 'doblar, articular', es decir, hacen alusión al movimiento articulatorio del codo, por esto es muy verosímil esta etimología. La forma hetita gipeššr tampoco está claramente estudiada y la única explicación verosímil es la de Čop, antes mencionada.

Finalmente, nos queda por examinar la forma griega ó $\gamma \kappa \omega$ $v$ 'codo'. Procede de la raíz ${ }^{*} H_{2} e n k$ - 'curvar, doblar', que se encuentra atestiguada en numerosas lenguas indoeuropeas: ai. añcati 'doblar, curvar', lat.

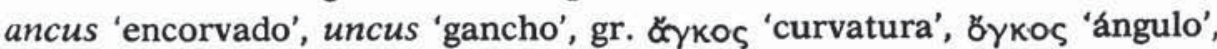
het. hink - 'hacer una reverencia', etc. ${ }^{99}$. En griego ó $\gamma \kappa \omega$ ' se aplica propiamente a la articulación de una extremidad vista desde fuera o desde dentro, y más concretamente al codo. Más tarde se produjo un desplazamiento semántico pasando a designar lo mismo que $\beta$ poxi $\omega_{\nu}$ en oposición a $\pi \hat{x} x u s$.

Con esto queda expuesto lo referente a las designaciones de la mano, el brazo y el codo en las lenguas indoeuropeas. Hemos ido viendo a lo largo del trabajo cómo, a menudo, las designaciones de ciertas partes del cuerpo, como son el brazo y el codo, han accedido a tales a partir de una idea más general que aludía a su función o a su condición de 'articulación', 'junta', etc. En el caso del codo no existe un vocablo primario, por así llamarlo, cuyo origen sea imposible de rastrear. Se trata siempre de creaciones lexicales a partir de raíces que significan 'doblar', 'torcer', etc., según hemos visto.

En el caso de 'brazo' nos encontramos con mayores dificultades. Por un lado, se han producido substituciones del hombro por el brazo, pasando la raíz que significa 'ajustar', caso de * $(H) e r H^{i}$, al brazo. Esto se ha producido en a. esl. con la forma ramo, rame que sirve igualmente para designar el hombro, o en a. irl. dōe 'brazo', emparentada con av. daoš 'parte superior del brazo, hombro', sct. doș- 'antebrazo'. La existencia de sinónimos en las lenguas tiene una explicación fácil. En principio, no se trata de sinónimos sino que cada una de las formas se aplica a realidades diferentes. Así, por ejemplo, gr. $\beta$ poxi $i \omega v$ designaba el antebrazo, en oposición a $\pi \hat{x} \chi \cup \varsigma$, aunque más tarde llegaron a

\footnotetext{
97 Riano en Pólux II 180.

98 Simaristo en Ateneo $399 \mathrm{~b}$, aunque por su aparición tardía es posible que se trate de una variante textual por kú $\mu$ ßos.

99 Van Windekens incluye en esta raíz toc. A ớkaläm, B orjkolmo 'elefante', cuyo primer elemento ờ $k$ - habría que relacionarlo con toc. A añcäl 'arco', de la misma raíz. Cf. Le tokharien confronté... I, p. 337.
} 
confundirse. En lat. armus se aplica generalmente a las extremidades delanteras de un animal y en muy pocas raras ocasiones al hombre, para el que se utiliza el término bracchium. Lo mismo sucede con el ai. ìrmá- frente a bāhú-. En a. irl. lām hace referencia tanto a 'mano' como a 'brazo'. Dado que la etimología de esta palabra está unida a la de la 'palma de la mano', hay que pensar que el nombre del brazo, o bien no existió o bien fue substituido por el de la mano. Hay que tener en cuenta que el otro término usado para designar el brazo, dōe, en origen designaba el hombro.

En eslavo, aparte de las ya mencionadas formas ramo, rame encontramos myšica 'brazo' que originariamente significaba 'ratoncillo' y de ahí pasó a significar 'músculo' como hemos dicho en páginas anteriores. Su creación responde a un fenómeno habitual en otras lenguas indoeuropeas y el paso de 'músculo' a 'brazo' pudo ser facilitado por la falta de precisión del término ramo.

Finalmente hay que hacer notar que las lenguas bálticas, a excepción del a. prus., utiliza la misma palabra para 'mano' y 'brazo': lit. rankd, let. ruoka, y lo mismo sucede en ruso moderno, ruká. Esto muestra que las oposiciones basadas en rasgos que la mente humana considera relevantes, pueden no serlo en lenguas diferentes a la nuestra. En griego moderno, por ejemplo, llaman brazo ' $\chi \varepsilon \hat{\varepsilon} \iota^{\prime}$ a toda la extremidad superior incluida la mano.

Las designaciones del brazo no son, pues, uniformes y no podemos, obviamente, reconstruir una única forma al indoeuropeo. Se trata de creaciones lexicales a partir de diferentes raíces. Por un lado de la raíz * $(H) e r H^{i-}$ 'ajustar' en diferentes grados apofónicos, que también sirve, en ocasiones, para designar el hombro, y a veces el codo. Ello hace verosímil pensar que en origen no se diferenciaba la extremidad superior en varias partes, como se hace en muchas lenguas actuales, sino que todo ello era un conjunto, incluida la mano. Cuando se especificó cada parte de la extremidad superior, las formas antiguas pasaron a veces a especializarse en usos más restringidos: patas de los animales, etcétera.

Las formas $\pi \hat{n} x \cup \varsigma$ y bähú- hemos de concluir que se trata de una innovación, no forzosamente reciente, del griego y el indo-iranio. No existen rastros fuera de estas lenguas, lo que hace sospechosa su pertenencia al indoeuropeo común. El toc. A poke, B pauke de la raíz *bheugh- 'doblar' se encuentra, asimismo, aislado. Sin embargo, su derivación es fácil de ver ya que existen paralelos en la extremidad inferior, la pierna, que también a veces deriva de esta noción de 'doblar', e incluso en ai. bhujá- 'brazo' de la misma raíz.

LIII, $2 .^{\circ}-5$ 
El codo, por su parte, deriva la mayoría de las veces de la raíz ${ }^{*} H_{3} l_{e H}{ }^{i_{2}}$ - 'doblar', pero las formas resultantes no se retrotraen a una sola palabra. Es decir, son todas ampliaciones de la mencionada raíz, pero cada lengua lo ha hecho de un modo diferente. Esto hace pensar que se trata de un término de creación reciente, a partir de una tendencia general de derivar las palabras que designan una articulación de raíces que significan 'doblar', 'articular', etc.

Por otra parte, hay que resaltar la frecuencia con que se intercambian la designación del codo y la del antebrazo, que lleva a que una sola forma signifique las dos cosas, así gr. $\omega \lambda \varepsilon \dot{\varepsilon} \eta$, lat. ulna, alb. lërë, a. prus. woaltis 'cúbito', 'antebrazo'. Esto nos da una idea de que la noción de 'doblar', inherente a la raíz, se pierde con el tiempo, caso de los compuestos germánicos que necesitan ser recaracterizados con la raíz *bheugh-.

En hetita no encontramos la palabra 'codo' derivada de esta raíz, pero sí formas relacionadas con ella, ya examinadas. El hetita gipešsar de etimología poco clara sería la excepción a la tendencia general de derivar la designación del codo de la noción de 'doblar'. Tendencia que

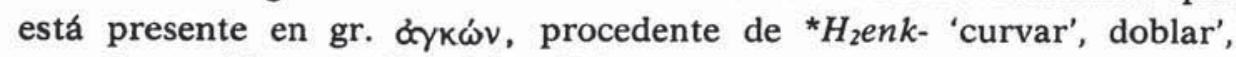
y en lat. cubitus de *keu-b/keuH-b- 'doblar'.

Nos queda hacer alusión a la designación de 'mano' que, a diferencia del codo y el brazo, no deriva de una noción determinada representada por una o varias raíces. Esto es debido a factores de variada índole. Por un lado, la multiplicidad de funciones de la mano que no nos permite asociarla únicamente con la idea de 'agarrar'. Por otro lado es muy probable que en algunos casos nos enfrentemos a verdaderos vocablos primarios sin derivación alguna de una raíz conocida. Aún así, la tendencia generalizada ha sido conectar siempre la etimología de las distintas designaciones de 'mano' a una raíz indoeuropea concreta. En ciertos casos esto es posible e incluso probable y así lo hemos hecho. En otros casos no lo es. Desde este punto de vista, diremos que la mano en indoeuropeo presenta derivados de las siguientes raíces: * gher- 'aga-

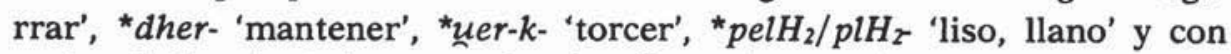
serias dudas, *mā- 'tocar, palpar' y *kem- 'agarrar'.

Las formas derivadas de "gher- son las más documentadas, y se encuentran en anatolio, por lo que consideramos a estas formas de gran antiguiedad. No obstante, no existen huellas de esta rafz ni en las lenguas germánicas, ni en las bálticas, eslavas ni, quizá, en latín. Lo mismo sucede con lat. manus que se encuentra reducida al área itálica y germánica. En este último grupo de lenguas, aparte de las formas emparentadas con lat. manus (aaa. munt, a. nórd. mund, etc.), existen 
otras derivadas de una hipotética raíz *kem- 'agarrar': aaa. hant, a. nórd. hond. La explicación de los dos grupos de formas podría estar en que aaa. munt, a. nórd. mund han pasado a significar 'protección, tutela', y han desarrollado un sentido jurídico, mientras que las otras han conservado el sentido originario ${ }^{100}$.

La diversidad de formas para designar la mano nos hace pensar en que realmente no debió existir una designación única en el indoeuropeo más antiguo, y sólo posteriormente se acuñaron unos términos para esta parte del cuerpo. Es curioso que la gran inestabilidad que hemos detectado en la designación de 'mano' haya cesado en el momento en que se produjo tal designación. Es decir, una vez que se fijó un término para 'mano', éste ha continuado, con las evoluciones precisas, hasta nuestros días sin más cambios. La comparación con otras partes del cuerpo, que sí están unánimemente representadas en todas las lenguas, indica que la designación de las partes de la extremidad superior del cuerpo humano careció de importancia frente a la de otros órganos como el corazón, la cabeza, la rodilla, el pie, etc.

Rosa PEDRero

100 Ya en pruebas este artículo, he tenido conocimiento del trabajo de T. L. Markey «The grammaticalization and institutionalization of Indo-European HAND*, JIES 12, 1984, pp. 261-292, donde se aborda el problema desde perspectivas interesantes. 